\title{
The Design of DC Electromagnetic Relay's Accelerated Life Test Equipment
}

\author{
HU Tongjiang, \\ Department of Vehicle \& Electronic Engineering, \\ Ordnance Engineering College, \\ Shijiazhuang 050003, China
}

\author{
YANG Fengbiao, \\ Department of Vehicle \& Electronic Engineering, \\ Ordnance Engineering College, \\ Shijiazhuang 050003, China
}

\author{
XU Yicheng \\ Department of Vehicle \& Electronic Engineering, \\ Ordnance Engineering College, \\ Shijiazhuang 050003, China
}

\begin{abstract}
The digitized constant current control system was designed according to the problems of small DC electromagnetic relay's contact test current, long test time, service life difficult to predict etc. This equipment can fulfill constant stress, step stress and sequence to stress accelerated life test of DC electromagnetic relay by adjust the current. The measure and control software based on Labview, realized test current continuous controlled, pressure drop of contact closed and disconnected automatic measurement, failure of automatic identification, action frequency controllable regulation, data automatic storage. Through the practical application, equipment has the characteristics of test accuracy, reliable work, etc. This equipment offer fast and effective test condition for accurately predicting DC electromagnetic relay's service life.
\end{abstract}

Keywords: DC Electromagnetic Relay; Accelerated Life Testing; Constant Current Control; System Design

\section{Introduction}

Relay is widely used in manufacturing, agriculture, transportation, national defense military, space technology and daily life. It is a basic component indispensable in the electronic equipment for remote control, remote sensing, communication, detection, and protection.
Whether relay can work normally will be directly related to the stability and reliability of the equipment or products containing it. With the development of relay technology and the increasingly serious relay application environment, the requirements for relay's performance, service life, and reliability are getting higher and higher [1]. Electromagnetic relay often fails to work at contacts and its failure modes are as follows:

First, contact resistance is composed of shrinkage resistance and membrane resistance. The contact surface at contacts is uneven and actually only a few contacts can be really touched, making contact resistance at contacts larger.

Second, an obvious arc will appear at contact's on-off moment if load current is large, making contact arc welding and contacts stick together.

Third, due to non-strict production process and the redundancy remaining on the surface of contacts, arc energy increase and arc time prolonging will be caused if arc between contacts burns, making the thermal ablation of cladding materials exacerbated and inducing contacts adhesion to fail.

Contact faults account for about $90 \%$ of all relay faults, among which fusion welding failure is one of the serious faults [2]. Studies have shown that the adhesion of relay contacts may occur in the contact closure process or contact breaking 
process [3], and DC electromagnetic relay's arc is more difficult to extinct than that of $\mathrm{AC}$ electromagnetic relay.

At present, the service life and other parameters of electromagnetic relay have been detected and evaluated by most production units of China using computer control system [4], but the experiment environment conditions are often under room temperature; contact on-off is often operated at rated current or lower current, and thus, a long test time is required to spend on its operation, and the prediction on the service life of contacts is very difficult under a bad environment [5]. To solve above problems, an accelerated life test for enlarging stress and shortening time was proposed in this paper.

\section{Hardware design}

\subsection{System design scheme}

DC electromagnetic relay's accelerated life test equipment included computer testing and control system, constant current control module, relay coil control circuit, test object interface and other parts, and its system principle was is shown in Fig.1.

Computer testing and control system includes Labview programmed measure and control software and 2 data acquisition cards produced by Art-control. For USB2816, AD acquisition was differentially input and input range was unipolar input $(0 \sim 10 \mathrm{~V})$; the cut-in voltage and sealing voltage with 16 pairs of contacts could be simultaneously tested because 2 acquisition cards were applied. 2 acquisition cards' 16-way DIO output was applied to control the on-off of test objects' coil, and 4-way analog output was used to set the output current of the constant current control module.

To reduce the output power and improve the working reliability, 4 independent constant current control circuit were applied, the output current of each circuit was set in 0-20A, and maximum output voltage was DC100V. When test object's contact was turned on, the maximum voltage between different contacts was $100 \mathrm{~V}$ (beyond the scope of the data acquisition card input), and thus, $100 \mathrm{~V} / 10 \mathrm{~V}$ voltage sensor was applied to isolating voltage transformation.

Coil control circuit was used to control the power on-off of test object's coil, and the coil's current loop was isolated from input signal for ensuring the reliability of the data acquisition and control.

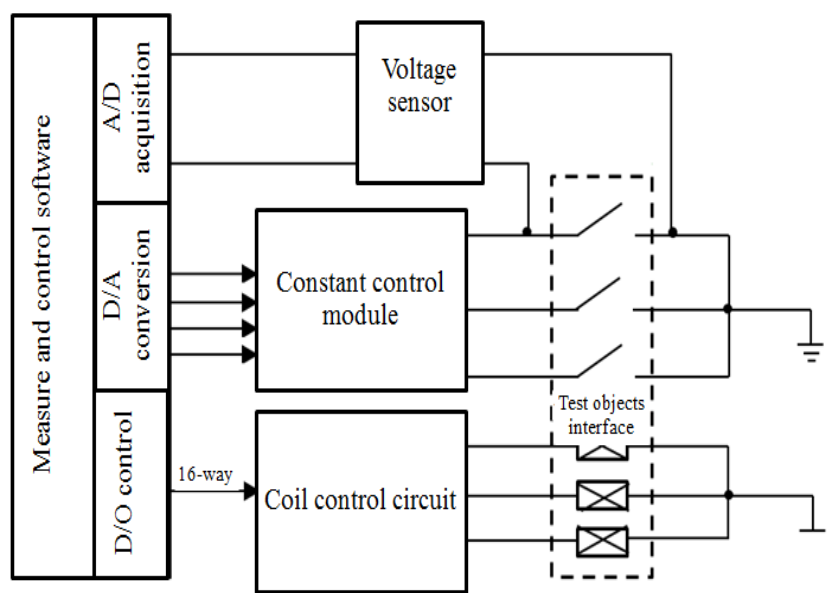

Fig.1 the functional block diagram of electromagnetic relay's accelerated life test equipment

\subsection{Constant current control module}

Constant current control module was composed of 4 independent constant current control circuits, and each control circuit was identical and the principle block diagram is shown in Fig. 2 .

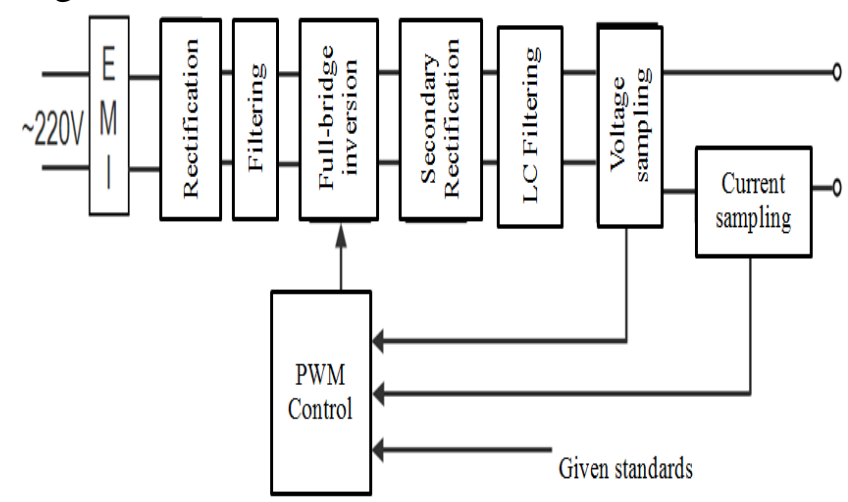

Fig.2 The functional block diagram of constant current control circuit

For control circuits, AC220V electric supply input was applied; uncontrollable full-bridge rectification was conducted via EMI filter, and 4 
parallel capacitor filters $(560 \mu \mathrm{F} / 450 \mathrm{~V})$ were used after bridge rectification, in order to get stable output DC. For full-bridge inverter circuit, 4 bridge arms were constituted by $25 \mathrm{~A} / 1200 \mathrm{~V}$ rapid IGBT, and RC buffer circuit was installed for each IGBT to absorb on-off peak. The output voltage could be $100 \mathrm{~V}$ to the maximum, and therefore, full-bridge inverter output end output via bridge secondary rectifier and LC filter and then was sent to PWM control circuit via voltage and current sampling circuits. For PWM control circuit, SG3525 was used as the main control chip and voltage sampling signal was sent to SG3525's soft start end, and SG3525's startup capacitor discharged under the control of voltage feedback signal and then restarted up if output voltage exceeded $100 \mathrm{~V}$, to ensure the output voltage not to overrun; current sampling signal was sent to the inverting input end of SG3525's error amplifier and controlled the duty cycle of SG3525's output PWM waveform in comparison with the given signal sent to the same-phase end of SG3525's error amplifier, so that the output current of the fullbridge inverter circuit was controlled, constant current was maintained. In this process, the reference signal was given by computer measure and control software via D/A conversion. Constant current control circuit worked under the double loop control mode (inner current loop and outer voltage loop).

\subsection{Coil control circuit}

Coil control circuit had 16 ways for controlling the coil on-off of 16 test objects, and the principle was shown in Fig.3. In Fig.3, switch signal was the control signal output by computer measure and control software via D/A conversion, which was added into the same end of comparator $\mathrm{U}_{2}$; to improve the antiinterference ability of control circuit, bleeder circuit (1:1) was composed of resistors R1 and $\mathrm{R} 2$, and partial pressure signal was input to the inverting end of comparator via the filtering of capacitor $\mathrm{C} 1$. $\mathrm{U}_{2}$ output high level, triode $\mathrm{Q}_{2}$ was conducting, and coupling $\mathrm{U}_{1}$ worked and its output end was conducting, and then the coil loop of test objects was switched on, if the control signal output by D/A conversion was about $5 \mathrm{~V} ; \mathrm{U}_{2}$ output low level, triode $\mathrm{Q}_{2}$ was shut off, and coupling output end and triode $\mathrm{Q}_{1}$ were cut off, and then coil loop of test objects was off. According to the state of switch signal, the cut-in voltage and sealing voltage of the contacts of test objects could be tested using measure and control software. In Fig.3, $\mathrm{V}_{\mathrm{d}}$ was coil voltage and kept independent of control signal's $5 \mathrm{~V}$ power supply, aiming to separate control circuit from executive circuit and improve the anti-interference capability.

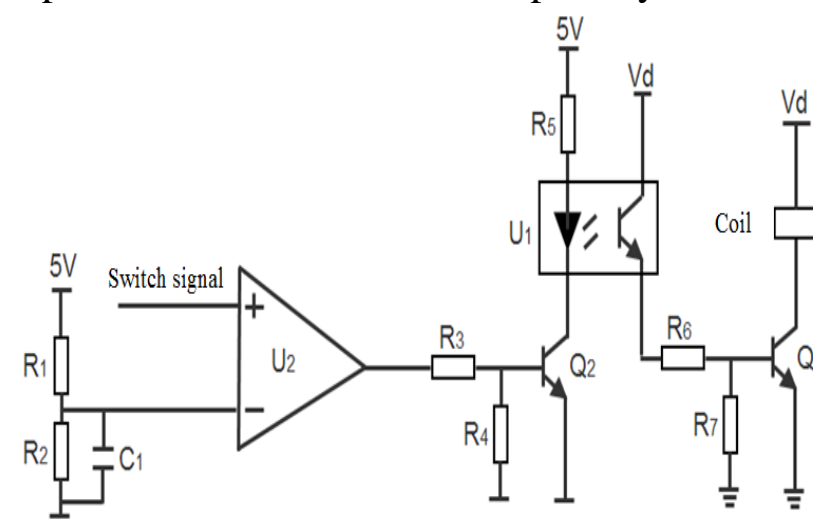

Fig. 3 the functional diagram of coil control circuit

\section{Software design}

In measure and control software, test object's coil on-off control, contact cut-in and closure and voltage drop measurement, the on-off frequency of switch and corresponding measured values were mainly completed, and whether test objects were damaged was judged according to the preset threshold value. Test objects were judged to fail if the measured data frequently exceeded the limiting value.

Test objects were judged to fail if any of the following circumstances occurred [6].

(1) Closing contact's contact voltage drop $U j$ exceeded the following limits:

a. Contact voltage drop's limit Ujm was 5\% or $10 \%$ of contact circuit's open-circuit voltage $U N$ 
b. Contact voltage drop's limit was $0.5 \mathrm{~V}$ if load current $=100 \mathrm{~mA}$, but $1 \mathrm{~V}$ if load current $=1 \mathrm{~A}$

(2) The voltage $U c$ between break contacts was lower than the limit $U c x$ ( $U c x$ was $90 \%$ of contact circuit's open-circuit voltage)

If test objects were judged to fail and USB2816's corresponding DIO output end was changed to zero level, the objects were blocked. The work flow of the measure and control software was shown in Fig.4.

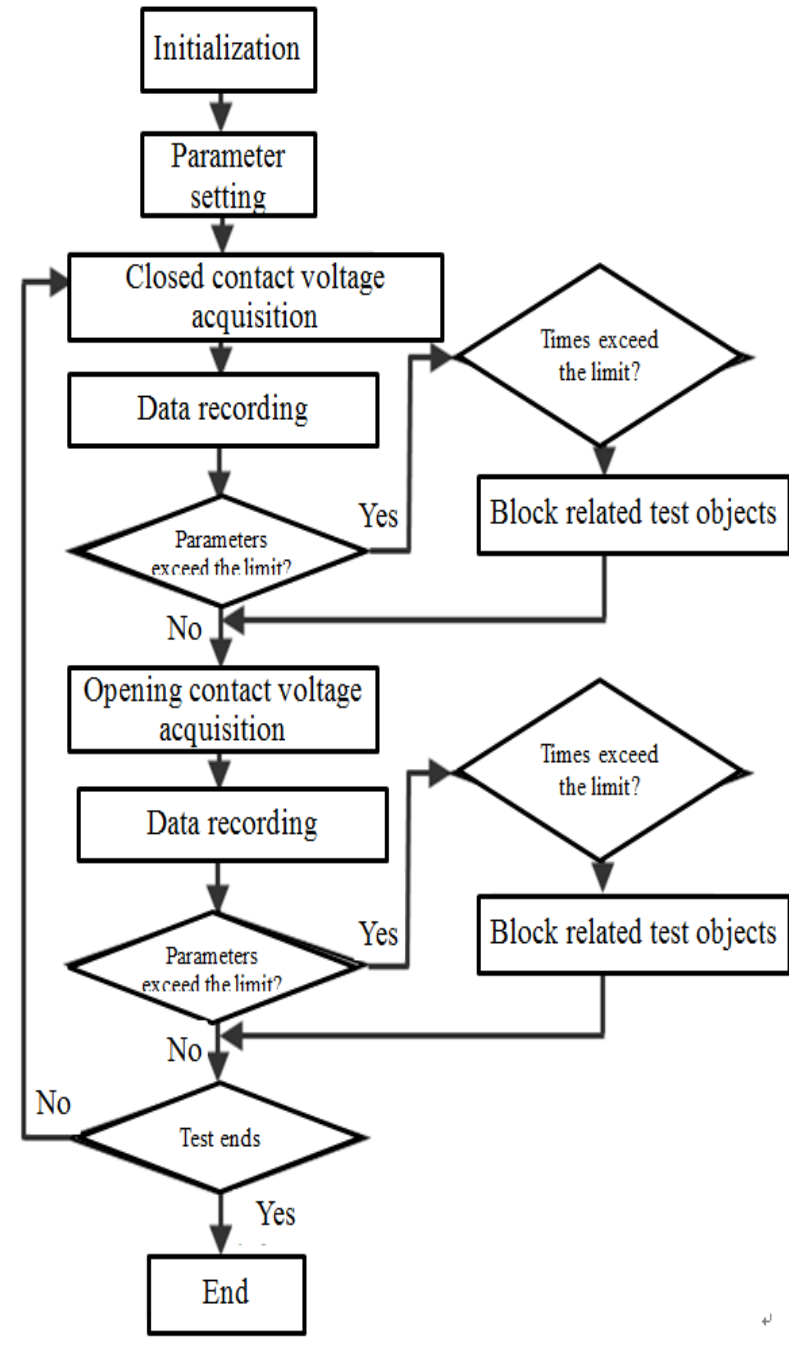

Fig.4 The flow chart of measurement and control

In initialization, 2 data acquisition cards' ID, data buffer, input and output range, and output current were set, and the input range of $\mathrm{A} / \mathrm{D}$ sampling was set to $0 \sim 10 \mathrm{~V}$ because the maximum voltage output by the current limit control module was $10 \mathrm{~V}$; according to the power supply voltage classes of coil control circuit, D/A output range was set to $0-5 \mathrm{~V}$. In the parameter settings, test objects' coil on-off frequency, contact voltage drop's threshold at contact closure and cut-in, the number of corresponding operations, and $\mathrm{d}$ overrun rat were set. Real data was difficult to acquire in sampling if coil's switch frequency was too high, but the work efficiency was affected if the frequency was too low, and therefore, the frequency was necessarily set according to test objects' switching characteristics and data acquisition cards' sampling rate. The number of corresponding operates and the overrun rate was used to detect the overrun quantity in the prescribed operating frequency: test objects were judge to fail and also were blocked if the preset number of corresponding operations was 10 and the overrun rate was $50 \%$, and 5 times of overrun in the latest 10 samplings occurredthis was used as a basis for measuring the contact voltage drop of contact closure and cutin. After every each data measurement, switch frequency and measurement data were stored in Excel. The test ended if all test objects failed.

\section{Conclusion}

According to the principle of accelerated life test, the continuous output of constant current system was controlled using digital technology. According to the specific experimental requirements, electromagnetic relays' on-off was tested under different current levels and different temperature and humidity environment requirements to meet the accelerated life test requirements of constant stress, step stress, and progressive stress. Meanwhile, relevant application software was applied using Labview. Thus, a rapid and effective test system was provided for accurately predicting the service life of electromagnetic relay. 


\section{References}

[1] YUAN Yuan, LUO Yanyan, LI Wenhua. Design of Electromagnetic Relay's Accelerated Life Test System [J]. Low Voltage Apparatus, 2006 (1): 50-52.

[2] Jiang Dongsheng, Zhang Pei, Liu Xinjun. The Contact Life of Spacecraft Relay under Inrush Current [J]. Spacecraft Environment Engineering, 2013, 30 (6): 623-626.

[3] Li Zhenbiao,Wu Xixiu, Wei Meifang. Influence of Short Arc on Contacts Dynamic Welding of Automotive Relay [J]. Low Voltage Apparatus, 2007 (1): 10-21.
[4] Lu Fangwei, Yu Hongjun. PC Testing Instrument for Current (Voltage) Relay [J]. Techniques of Automation and Applications, 2003, 22 (3): 60-63.

[5] Yu Haichang. The software design of PC instrument for relay detecting $[\mathrm{J}]$. Information Technology, 2003, 27 (2): 8182.

[6] Jianguo LIU, Yiliang Tang. Electrical Reliability Theory and Its Application [M]. Machinery Industry Press, 1996.10. 